cPA decreases plasma cholesterol

\title{
Short-term treatment with a 2-carba analog of cyclic phosphatidic acid induces lowering of plasma cholesterol levels in ApoE-deficient mice
}

Tamotsu Tsukahara $^{\mathrm{a}^{*}}$, Hisao Haniu ${ }^{\mathrm{b}}$, Yoshikazu Matsuda $^{\mathrm{c}}$, and Kimiko Murakmi-Murofushi ${ }^{\mathrm{d}}$

${ }^{\mathrm{a}}$ Department of Molecular Pharmacology and Neuroscience, Nagasaki University Graduate School of Biomedical Sciences, 1-14 Bunkyo-machi, Nagasaki 852-8521, Japan

${ }^{\mathrm{b}}$ Institute for Biomedical Sciences, Shinshu University Interdisciplinary Cluster for Cutting Edge

Research 3-1-1 Asahi, Matsumoto, Nagano 390-8621, Japan

${ }^{\mathrm{c} C l i n i c a l}$ Pharmacology Educational Center, Nihon Pharmaceutical University, Ina-machi, Saitama 362-0806, Japan

${ }^{\mathrm{d}}$ Endowed Research Division of Human Welfare Sciences, Ochanomizu University, 2-1-1, Ohtsuka, Bunkyo-ku, Tokyo 112-8610, Japan

Running title: cPA decreases plasma cholesterol

To whom correspondence should be addressed: Tamotsu Tsukahara, Department of Molecular

Pharmacology and Neuroscience, Nagasaki University Graduate School of Biomedical Sciences, 1-14

Bunkyo-machi, Nagasaki 852-8521, Japan

E-mail: $\underline{\text { ttamotsu@nagasaki-u.ac.jp }}$

Tel: $+81-75-852-2473$

Fax: $+81-75-852-2420$ 


\begin{abstract}
Plasma cholesterol levels are associated with an increased risk of developing atherosclerosis. An elevated low-density lipoprotein cholesterol (LDL-C) level is a hallmark of hypercholesterolemia in metabolic syndrome. Our previous study suggested that when acetylated LDL (AC-LDL) was co-applied with a PPAR $\gamma$ agonist, rosiglitazone (ROSI), many oil red O-positive macrophages could be observed. However, addition of cyclic phosphatidic acid (cPA) to ROSI-stimulated macrophages completely abolished oil red O-stained cells, indicating that cPA inhibits PPAR $\gamma$-regulated AC-LDL uptake. This study aimed to determine whether metabolically stabilized cPA, in the form of a carba-derivative of cPA (2ccPA), could reduce plasma cholesterol levels and affect the expression of genes related to atherosclerosis in apolipoprotein E-knockout $\left(\mathrm{apoE}^{-/-}\right)$mice. 2ccPA reduced LDL-C levels in these mice $(\mathrm{n}=3)$ from 460 to $330 \mathrm{mg} / \mathrm{ml}$, from 420 to $350 \mathrm{mg} / \mathrm{ml}$, and 420 to $281 \mathrm{mg} / \mathrm{ml}$ under a western-type diet. $2 \mathrm{ccPA}$ also reduced expression of lipid metabolism-related genes, cytokines, and chemokines in ApoE-deficient mice on a high-fat diet. Taken together, these results suggest that 2ccPA governs anti-atherogenic activities in the carotid arteries of apoE-deficient mice.
\end{abstract}

Keywords: atherosclerosis; cholesterol; cyclic phosphatidic acid; high-density lipoprotein cholesterol (HDL-C); low-density lipoprotein cholesterol (LDL-C) 


\section{Introduction}

Atherosclerosis is a complex chronic vascular disease characterized by accumulation of plasma-derived lipids in arteries [1]. Modified lipoproteins, such as oxidized low-density lipoproteins (oxLDLs), which are generated in the vessel wall, up-regulate the expression of cytokines and chemokines $[2,3]$. These molecules attract lymphocytes and monocytes. In the arterial intima, immune cells generate various cytokines and chemokines, leading to a cascade of events, involving recruitment of additional inflammatory cells, further lipid accumulation, and increased migration, differentiation, and proliferation of smooth muscle cells (SMCs). Low-density lipoprotein (LDL) is a transporter of phospholipids and cholesterol in the blood [4].

One such phospholipid, cyclic phosphatidic acid (cPA), with a unique cyclic phosphate ring at the sn-2 and sn-3 positions of its glycerol backbone, is an analog of the growth factor-like phospholipid lysophosphatidic acid (LPA), and is a therapeutic a target for many diseases, including atherosclerosis [5], obesity [6], cancer [7, 8], and inflammation [9] [10]. Our previous in vitro study suggested that the suppressive effects of cPA on the growth and migration of human coronary artery endothelial cells (HCAECs) obtained from donors with type 2 diabetes (D-HCAECs) require the inhibition of peroxisome proliferator-activated receptor gamma $(\operatorname{PPAR} \gamma)$. cPA, along with a PPAR $\gamma$ antagonist, also prevented neointima formation, adipocyte differentiation, lipid accumulation, and downregulation of PPAR $\gamma$-target gene transcription in mouse macrophages [5]. Furthermore, activation of the endothelium by alkyl-LPA (AGP) has been implicated in the development of atherosclerosis, while VEGF production was elicited by AGP in D-HCAECs that express high PPAR $\gamma$ levels [11]. AGP is present in blood plasma [12] and its levels increase after minimal oxidative modification of LDL. High levels of AGP are also found in the lipid-rich necrotic core of human carotid artherosclerotic plaques. Interestingly, AGP increases the expression and secretion of interleukin (IL)-6 in HCAECs [11], but this effect is inhibited when HCAECs are co-treated with cPA [13].

Although atherosclerosis is not a novel characteristic of ApoE-deficient humans, the ApoE-knockout mouse is a well-established genetic animal model of atherosclerosis and has been used 
widely in studies of the effect of foods and drugs [14]. ApoE-deficient mice that are fed diets high in fat and cholesterol show markedly accelerated plaque development. In this study, we investigated the involvement of lipid metabolism-related as well as inflammation-related genes in carotid arteries of these mice, after treatment with or without a metabolically stabilized derivative of cPA, viz., 2-carba-cPA (2ccPA).

\section{Materials and methods}

\subsection{Materials}

2ccPA (18:1) was a kind gift from Kimiko Murakami-Murofushi, from Ochanomizu University, Tokyo Japan. A Histone deacetylase (HDAC) antibody sampler kit (\#9928S) was purchased from Cell Signaling Technology, Inc. (Beverly, MA, USA).

\subsection{Animals and treatment}

All animal experiments were performed in collaboration with Unitech Co., Ltd. (Chiba, Japan). BALB/c. KOR/SteSlc-Apoe ${ }^{\text {shl }}$ mice (6-week-old), weighing 20-25 g, were purchased from Japan SLC, Inc. (Shizuoka, Japan). The animals were kept in a humidity-controlled room on a 12-h light-dark cycle with standard food and water available ad libitum for 1 week. The mice were then divided randomly into two groups, with four animals in each group, and were fed a high-fat diet (D12108C, 1.25\% cholesterol),

purchased from Research Diet Inc. (New Brunswick, NJ, USA), for 4 weeks. The $\mathrm{ApoE}^{-/-}+2 \mathrm{ccPA}$ group were administered $2 \mathrm{ccPA}(5 \mathrm{~mL} / \mathrm{kg}$ bodyweight, $2 \mathrm{mg} / \mathrm{mL}$ in phosphate-buffered saline [PBS]) by subcutaneous injection, while the control group (ApoE $\mathrm{E}^{-/-}$group) was given an equal volume of PBS, for 4 weeks.

\subsection{Measurement of cholesterol and triglyceride levels}


The plasma levels of triglycerides (TGs), LDL cholesterol (LDL-C), high-density lipoprotein cholesterol (HDL-C), and total cholesterol (T-CHO) were measured in collaboration with Unitech Co., Ltd. (Chiba, Japan).

\subsection{Mouse carotid artery harvesting}

The carotid arteries of the mice were harvested 14 days after commencing the high fat diet in collaboration with Unitech Co., Ltd. (Chiba, Japan), and total RNA and protein was extracted using TRIzol reagent (Life Technologies, Carlsbad, CA, USA).

\subsection{Quantitative real-time PCR analysis}

The total RNA obtained from the carotid arteries was purified using a NucleoSpin RNA II kit (TaKaRa, Otsu, Japan), according to the manufacturer's protocol. Of this, $0.1 \mu \mathrm{g}$ was used for the subsequent synthesis of cDNA using a ReverTra Ace qPCR RT kit (Toyobo, Osaka, Japan), as recommended by the manufacturer. The levels of mRNA were determined with an ECO Real-Time PCR system (Illumina, Inc., San Diego, CA, USA), using SYBR Green Real-Time PCR Master Mix-Plus (Toyobo) and the following primer pairs: mCD36, 5'-TCCAGCCAATGCCTTTGC-3' (forward) and 5'-TGGAGATTACTTTTTCAGTGCAGAA-3' (reverse); FABP4, 5'-GGGAACCTGGAAGCTTGTCT-3' (forward) and 5'-ACTCTCTGACCG GATGGTGA-3' (reverse); PPAR $\gamma$, 5'-CCACCAACTTCGGAATCAGCT-3' (forward) and 5'-TTTGTGGATCCGGCAGTTAAGA-3' (reverse); IL-6, 5'-CTGCAAGAGACTTCCATCCAG-3' (forward) and 5'-AGTGGTATAGACAGGTCTGTTGG-3' (reverse); RANTES, 5'-ATATGGCTCGGACACCACTC-3' (forward) and 5'-CCTTCGAGTGACAAACACGA-3' (reverse); MCP-1, 5'-ATCCCAATGAGTAGGCTGGAGAGC-3' (forward) and 5'-CAGAAGTGCTTGAGGTGGTTGTG-3' (reverse); and $\beta$-actin, 5'-AGGCACCAGGGCGTGAT-3' (forward) and 5'-GCCCACATAGGAATCCTTCTGAC-3' (reverse). The specificity of the PCR amplifications was verified by melting curve analysis. Levels of expression were normalized to the 
endogenous reference gene encoding $\beta$-actin using the relative quantitative method $(\Delta \Delta \mathrm{Ct})$, as previously reported [15].

\subsection{Statistical analysis}

Student's $t$-test was used for statistical comparisons and $\mathrm{P}<0.05$ was considered statistically significant.

\section{Results and discussion}

During the progression of atherosclerosis, LDL accumulates within the macrophages and monocytes present in the intima [16]. In this study, plasma lipoprotein levels were determined after ApoE-deficient mice were fed a high-fat diet for 4 weeks (Fig. 1B). The average plasma T-CHO in the control high-fat diet group was $1756.7 \mathrm{mg} / \mathrm{mL}$, and 2ccPA treatment caused an $11 \%$ reduction, to $1556.7 \mathrm{mg} / \mathrm{mL}$ (Fig. 1C). Plasma TG levels were not different between the control and 2ccPA-treated mice after feeding them a high-fat diet for 4 weeks (Fig. 1D). However, the average LDL-C levels were reduced from 433.3 $\mathrm{mg} / \mathrm{mL}$ to $320.3 \mathrm{mg} / \mathrm{mL}$ (a $23 \%$ reduction; Fig. 1E) by 2ccPA treatment for 4 weeks, and HDL-C levels were increased in mice fed a high-fat diet and treated with 2 ccPA, from $17.3 \mathrm{mg} / \mathrm{mL}$ to $19.7 \mathrm{mg} / \mathrm{mL}$ (Fig. 1F). These results suggested that treatment with $2 \mathrm{ccPA}$ at a dose of $2 \mathrm{mg} / \mathrm{mL}$ for 4 weeks was significantly more effective than vehicle control.

Based on our previous study [6], we hypothesized that exogenous application of 2ccPA in vivo, should mitigate atherogenic progression in carotid arteries. In the present study, we selected five atherogenic target genes (Cd36, Pparg, Fabp4, and Vegf) and four cytokine- and chemokine-related genes (Il6, Rantes, Mcpl, and Tnfa), and monitored their mRNA expression level in the carotid arteries of mice with or without $2 c \mathrm{PA}$ treatment after 4 weeks on a high-fat diet. We previously identified these selected target genes from an in vitro experiment $[5,11]$.

As shown in Fig. 2B-D, we found that, in ApoE-deficient mice, the expression of the target genes was significantly decreased compared with the control group. 
Macrophages are thought to play many roles in atherogenic progression, including scavenging of trapped lipoproteins and cytokines [17]. CD36 is a scavenger receptor that plays a role in cytokine-induced macrophage fusion [18].

Moreover, our previous study suggested that stimulation of macrophages with the TZD drug, ROSI, elicits direct activation of PPAR $\gamma$-regulated genes and that cPA completely abolishes the transcriptional regulation of these genes by ROSI [5]. Furthermore, to examine the regulatory role of cPA on PPAR $\gamma$-dependent pathophysiological responses, we utilized a rodent model of vascular remodeling [5]. An LPA analog, AGP, induced neointima formation when applied topically within the carotid artery [19]. PPAR $\gamma$ is a member of the nuclear receptor superfamily that is involved in the regulation of lipid metabolism, glucose homeostasis, and cell differentiation [20, 21]. Neointima formation requires PPAR $\gamma$, because it is abolished by the PPAR $\gamma$ antagonist GW9662 and is absent in mice that are conditionally deficient in PPAR $\gamma$ in the cells of the vessel wall [5].

Fatty-acid-binding protein 3 (FABP3) is one of nine known cytosolic FABPs that range in size from 14 to $15 \mathrm{kDa}$. It is highly expressed in heart and skeletal muscle, as well as in other tissues (ref). Our previous study suggested that FABP3 is an LPA-binding protein expressed in HCAECs [22]. We have also demonstrated that FABP3 is translocated from the cytosol to the nucleus in response to LPA-mediated PPAR $\gamma$ activation. FABP appears to play a role in shuttling fatty acid ligands to the nucleus, where the ligands are released and bind to PPAR $\gamma$ [22].

VEGF is an important angiogenic factor reported to induce migration and proliferation of endothelial cells, and to enhance vascular permeability [23] [24]. A recent study has suggested that VEGF alters the rate of atherosclerotic plaque development. Celletti et al. reported that VEGF significantly increased macrophage levels in bone marrow and peripheral blood and increased the plaque area in cholesterol-fed rabbits [25]. As expected from our previous in vitro experiment [11], these results are consistent with the hypothesis that exogenously applied 2ccPA influences vascular wall responses in vivo.

Cytokines and chemokines have also been implicated in the pathogenesis of atherosclerosis, which is an inflammatory disease of the arterial wall [17]. Therefore, we also analyzed the expression of 
inflammatory mediators in ApoE-deficient mice on a high-fat diet, with or without 2ccPA treatment. As shown in Fig. 3A, B, C, and D, our results were consistent with the hypothesis that exogenously applied 2ccPA can effectively modulate vascular wall responses and inflammation in vivo.

Cardiovascular disease has been shown to trigger chronic inflammatory processes, and pro-inflammatory cytokines, such as IL-6, are important mediators of endothelial dysfunction in coronary artery disease [26]. Furthermore, in vivo and in vitro investigations have shown that various chemokines activate endothelial cells and inflammatory cells [17]. One of these chemokines, monocyte chemo-attractant protein-1 (MCP-1), has been detected in atherosclerotic lesions [27].

Earlier studies have shown that PPAR $\gamma$, which plays a key role in the cardiovascular system, is expressed in all cell types of the blood-vessel wall and in monocytes and macrophages [16]. Coronary artery disease is the most common type of heart disease, and the cardiovascular-related mortality typically involves atherosclerosis [26]. Our results thus raise the possibility of using cPA as a lead compound for the development of new treatments that act on PPAR $\gamma$. The migration of endothelial cells contributes to diverse aspects of vascular physiology, such as the development of atherosclerosis. Currently, cPA is considered to play a role in the anti-neointima activity of carotid arteries [5]. A previous report has demonstrated that cPA inhibited AGP-induced expression of class I histone deacetylases (HDACs, namely HDAC1, HDAC2, HDAC3, and HDAC8), which may affect subsequent transcriptional activity of target genes. A recent study also reported that high levels of AGP accumulate in atherosclerotic lesions in rodents model [16]. Furthermore, increased AGP serum levels are an important risk factor for atherosclerosis, and AGP accumulation in the vessel wall has been suggested to trigger neointima formation [5].

Our previous study has shown that human coronary artery endothelial cells (HCAECs) express high levels of HDAC2 and low levels HDAC1, HDAC3, and HDAC8. HDACs, which are known to activate PPAR $\gamma$ and enhance the expression of its target genes [13], have well-known functions in the regulation of chromatin structure and gene transcription via interactions with nuclear receptor co-repressors, such as SMRT and nuclear receptor corepressor (NcoR) [5]. HDAC2 plays critical roles in 
a wide range of biological events, mainly by means of its catalytic activity in removing acetyl groups from histones as well as non-histone proteins [28]. However, several independent investigations have pointed out that class I HDACs participate in chronic inflammation and vascular remodeling. HDAC2 is required for a major mechanism through which activated glucocorticoid receptors block transcription of inflammation related genes [29]. HDAC3 has been reported to inhibit PPAR $\gamma$ and nuclear transcription factor- $\kappa \mathrm{B}(\mathrm{NF}-\mathrm{\kappa B})$ [30] and HDAC3 inhibition restores PPAR $\gamma$ function in obesity [31]. The inflammatory process involves activation of a variety of signaling pathways and expression of many target genes, which may be mediated by PPAR $\gamma$ [32]. As shown in Fig. 4A, reduction of HDAC2 is believed to result from AGP mediated PPAR $\gamma$ activation in coronary artery in mice. We found that Hdac2 mRNA was expressed at high levels after treatment with 2ccPA for 28 days, while Hdac3 was expressed at low levels. We therefore hypothesize that PPAR $\gamma$ inhibition prevents development of atherosclerosis and that anti-atherosclerotic effects of 2ccPA are dependent of serum cholesterol levels.

Taken together, the results of this in vivo study indicated a relationship between 2ccPA and blood cholesterol levels, and supported the conclusions drawn from our previous in vitro study.

Future studies employing HDAC2 transgenic animals will further clarify the role of the cPA-HDAC2 axis in atherogenesis in vivo, and should shed more light on to therapeutic strategies against atherosclerosis.

\section{Acknowledgments}

This work was supported by a Grant-in-Aid for Scientific Research (B) (25293274 to Tamotsu Tsukahara) from the Japan Society for the Promotion of Science (JSPS) KAKENHI. 


\section{Author contributions}

T.T., Y.M., and H.H. conceived and designed the project; T.T. acquired the data; T.T. and H.H. analyzed and interpreted the data; and T.T. wrote the article. All authors read and approved the final version of the article.

\section{Author disclosure statement}

No competing financial interests exist. 


\section{References}

1. Ross R. The pathogenesis of atherosclerosis: a perspective for the 1990s. Nature. 1993;362(6423):801-9. doi: 10.1038/362801a0. PubMed PMID: 8479518.

2. Ross R. Atherosclerosis--an inflammatory disease. N Engl J Med. 1999;340(2):115-26. doi: 10.1056/NEJM199901143400207. PubMed PMID: 9887164.

3. Libby P, Ridker PM, Maseri A. Inflammation and atherosclerosis. Circulation. 2002;105(9):1135-43. PubMed PMID: 11877368.

4. Badimon L, Vilahur G. LDL-cholesterol versus HDL-cholesterol in the atherosclerotic plaque: inflammatory resolution versus thrombotic chaos. Ann N Y Acad Sci. 2012;1254:18-32. doi: 10.1111/j.1749-6632.2012.06480.x. PubMed PMID: 22548566.

5. Tsukahara T, Tsukahara R, Fujiwara Y, Yue J, Cheng Y, Guo H, et al. Phospholipase D2-dependent inhibition of the nuclear hormone receptor PPARgamma by cyclic phosphatidic acid. Mol Cell. 2010;39(3):421-32. doi: 10.1016/j.molcel.2010.07.022. PubMed PMID: 20705243; PubMed Central PMCID: PMC3446787.

6. Tsukahara T, Hanazawa S, Murakami-Murofushi K. Cyclic phosphatidic acid influences the expression and regulation of cyclic nucleotide phosphodiesterase 3B and lipolysis in 3T3-L1 cells. Biochem Biophys Res Commun. 2011;404(1):109-14. doi: 10.1016/j.bbrc.2010.11.076. PubMed PMID: 21095182.

7. Tsukahara T, Hanazawa S, Kobayashi T, Iwamoto Y, Murakami-Murofushi K. Cyclic phosphatidic acid decreases proliferation and survival of colon cancer cells by inhibiting peroxisome proliferator-activated receptor gamma. Prostaglandins Other Lipid Mediat. 2010;93(3-4):126-33. doi: 10.1016/j.prostaglandins.2010.09.002. PubMed PMID: 20932931.

8. Tsukahara T, Matsuda Y, Haniu H. Cyclic phosphatidic acid stimulates cAMP production and inhibits growth in human colon cancer cells. PLoS One. 2013;8(11):e81139. Epub 2013/11/28. doi: 10.1371/journal.pone.0081139

PONE-D-13-25966 [pii]. PubMed PMID: 24282571; PubMed Central PMCID: PMC3839875. 
9. Gotoh M, Nagano A, Tsukahara R, Murofushi H, Morohoshi T, Otsuka K, et al. Cyclic phosphatidic acid relieves osteoarthritis symptoms. Mol Pain. 2014;10:52. Epub 2014/08/16. doi: 1744-8069-10-52 [pii]

10.1186/1744-8069-10-52. PubMed PMID: 25123228; PubMed Central PMCID: PMC4141741.

10. Maeda-Sano K, Gotoh M, Morohoshi T, Someya T, Murofushi H, Murakami-Murofushi K.

Cyclic phosphatidic acid and lysophosphatidic acid induce hyaluronic acid synthesis via CREB transcription factor regulation in human skin fibroblasts. Biochim Biophys Acta. 2014;1841(9):1256-63. Epub 2014/05/23. doi: S1388-1981(14)00095-X [pii]

10.1016/j.bbalip.2014.05.004. PubMed PMID: 24845645.

11. Tsukahara T, Tsukahara R, Haniu H, Matsuda Y, Murakami-Murofushi K. Cyclic phosphatidic acid inhibits the secretion of vascular endothelial growth factor from diabetic human coronary artery endothelial cells through peroxisome proliferator-activated receptor gamma. Mol Cell Endocrinol. 2015;412:320-9. doi: 10.1016/j.mce.2015.05.021. PubMed PMID: 26007326.

12. Haseruck N, Erl W, Pandey D, Tigyi G, Ohlmann P, Ravanat C, et al. The plaque lipid lysophosphatidic acid stimulates platelet activation and platelet-monocyte aggregate formation in whole blood: involvement of P2Y1 and P2Y12 receptors. Blood. 2004;103(7):2585-92. doi:

10.1182/blood-2003-04-1127. PubMed PMID: 14645014.

13. Tsukahara T, Haniu H, Matsuda Y. Cyclic phosphatidic acid inhibits

alkyl-glycerophosphate-induced downregulation of histone deacetylase 2 expression and suppresses the inflammatory response in human coronary artery endothelial cells. Int J Med Sci. 2014;11(9):955-61. doi: 10.7150/ijms.9316. PubMed PMID: 25013374; PubMed Central PMCID: PMC4081316.

14. Meir KS, Leitersdorf E. Atherosclerosis in the apolipoprotein-E-deficient mouse: a decade of progress. Arterioscler Thromb Vasc Biol. 2004;24(6):1006-14. doi:

10.1161/01.ATV.0000128849.12617.f4. PubMed PMID: 15087308. 
15. Tsukahara T, Haniu H, Matsuda Y. PTB-associated splicing factor (PSF) is a PPARgamma-binding protein and growth regulator of colon cancer cells. PLoS One. 2013;8(3):e58749. doi: 10.1371/journal.pone.0058749. PubMed PMID: 23516550; PubMed Central PMCID: PMC3596311. 16. Zhang C, Baker DL, Yasuda S, Makarova N, Balazs L, Johnson LR, et al. Lysophosphatidic acid induces neointima formation through PPARgamma activation. J Exp Med. 2004;199(6):763-74. doi: 10.1084/jem.20031619. PubMed PMID: 15007093; PubMed Central PMCID: PMC2212723.

17. Osterud B, Bjorklid E. Role of monocytes in atherogenesis. Physiol Rev. 2003;83(4):1069-112. doi: 10.1152/physrev.00005.2003. PubMed PMID: 14506301.

18. Helming L, Winter J, Gordon S. The scavenger receptor CD36 plays a role in cytokine-induced macrophage fusion. J Cell Sci. 2009;122(Pt 4):453-9. doi: 10.1242/jcs.037200. PubMed PMID: 19155290; PubMed Central PMCID: PMC2714432.

19. Tsukahara T, Tsukahara R, Yasuda S, Makarova N, Valentine WJ, Allison P, et al. Different residues mediate recognition of 1-O-oleyllysophosphatidic acid and rosiglitazone in the ligand binding domain of peroxisome proliferator-activated receptor gamma. J Biol Chem. 2006;281(6):3398-407. Epub 2005/12/03. doi: M510843200 [pii] 10.1074/jbc.M510843200. PubMed PMID: 16321982.

20. Marx N, Sukhova G, Murphy C, Libby P, Plutzky J. Macrophages in human atheroma contain PPARgamma: differentiation-dependent peroxisomal proliferator-activated receptor gamma(PPARgamma) expression and reduction of MMP-9 activity through PPARgamma activation in mononuclear phagocytes in vitro. Am J Pathol. 1998;153(1):17-23. Epub 1998/07/17. doi: S0002-9440(10)65540-X [pii]. PubMed PMID: 9665460; PubMed Central PMCID: PMC1852950. 21. Tontonoz P, Spiegelman BM. Fat and beyond: the diverse biology of PPARgamma. Annu Rev Biochem. 2008;77:289-312. Epub 2008/06/04. doi: 10.1146/annurev.biochem.77.061307.091829. PubMed PMID: 18518822. 
22. Tsukahara R, Haniu H, Matsuda Y, Tsukahara T. Heart-type fatty-acid-binding protein (FABP3) is a lysophosphatidic acid-binding protein in human coronary artery endothelial cells. FEBS Open Bio. 2014;4:947-51. Epub 2014/11/27. doi: 10.1016/j.fob.2014.10.014 S2211-5463(14)00103-X [pii]. PubMed PMID: 25426414; PubMed Central PMCID: PMC4239478.

23. Inoue M, Itoh H, Ueda M, Naruko T, Kojima A, Komatsu R, et al. Vascular endothelial growth factor (VEGF) expression in human coronary atherosclerotic lesions: possible pathophysiological significance of VEGF in progression of atherosclerosis. Circulation. 1998;98(20):2108-16. Epub 1998/11/17. PubMed PMID: 9815864.

24. Hoeben A, Landuyt B, Highley MS, Wildiers H, Van Oosterom AT, De Bruijn EA. Vascular endothelial growth factor and angiogenesis. Pharmacol Rev. 2004;56(4):549-80. Epub 2004/12/17. doi: $56 / 4 / 549$ [pii]

10.1124/pr.56.4.3. PubMed PMID: 15602010.

25. Celletti FL, Waugh JM, Amabile PG, Brendolan A, Hilfiker PR, Dake MD. Vascular endothelial growth factor enhances atherosclerotic plaque progression. Nat Med. 2001;7(4):425-9. Epub 2001/04/03. doi: 10.1038/86490

86490 [pii]. PubMed PMID: 11283668.

26. Tousoulis D, Charakida M, Stefanadis C. Endothelial function and inflammation in coronary artery disease. Heart. 2006;92(4):441-4. doi: 10.1136/hrt.2005.066936. PubMed PMID: 16159981; PubMed Central PMCID: PMC1860901.

27. Tanaka E, Shimokawa H, Kamiuneten H, Eto Y, Matsumoto Y, Morishige K, et al. Disparity of MCP-1 mRNA and protein expressions between the carotid artery and the aorta in WHHL rabbits: one aspect involved in the regional difference in atherosclerosis. Arterioscler Thromb Vasc Biol. 2003;23(2):244-50. PubMed PMID: 12588766.

28. Heusch G, Libby P, Gersh B, Yellon D, Bohm M, Lopaschuk G, et al. Cardiovascular remodelling in coronary artery disease and heart failure. Lancet. 2014;383(9932):1933-43. doi: 10.1016/S0140-6736(14)60107-0. PubMed PMID: 24831770; PubMed Central PMCID: PMC4330973. 
29. Ito K, Barnes PJ, Adcock IM. Glucocorticoid receptor recruitment of histone deacetylase 2 inhibits interleukin-1beta-induced histone $\mathrm{H} 4$ acetylation on lysines 8 and 12. Mol Cell Biol. 2000;20(18):6891-903. PubMed PMID: 10958685; PubMed Central PMCID: PMC88765.

30. Gao Z, He Q, Peng B, Chiao PJ, Ye J. Regulation of nuclear translocation of HDAC3 by IkappaBalpha is required for tumor necrosis factor inhibition of peroxisome proliferator-activated receptor gamma function. J Biol Chem. 2006;281(7):4540-7. doi: 10.1074/jbc.M507784200. PubMed PMID: 16371367; PubMed Central PMCID: PMC1447600.

31. Ye J. Improving insulin sensitivity with HDAC inhibitor. Diabetes. 2013;62(3):685-7. doi: 10.2337/db12-1354. PubMed PMID: 23431009; PubMed Central PMCID: PMC3581218.

32. Duan SZ, Usher MG, Mortensen RM. Peroxisome proliferator-activated receptor-gamma-mediated effects in the vasculature. Circ Res. 2008;102(3):283-94. Epub 2008/02/16. doi: 102/3/283 [pii]

10.1161/CIRCRESAHA.107.164384. PubMed PMID: 18276926. 


\section{Figure legends}

Figure 1. Effect of 2ccPA on plasma cholesterol and lipoprotein profiles in $\mathrm{ApoE}^{-/-}$mice. (A) Chemical structure of 2ccPA (18:1). (B) Schematic showing the protocol for dosing schedule in ApoE ${ }^{-/-}$mice. (C) 2ccPA significantly decreases total cholesterol levels $(\mathrm{P}=0.01$ versus non-treated mice). (D) Plasma triglyceride (TG) levels were not different between the control and 2ccPA-treated mice fed a high-fat diet for 4 weeks. (E) LDL-C ( $\mathrm{P}=0.01$ versus non-treated mice) in $\mathrm{ApoE}^{-/-}$mice fed with high-fat diet. (F) 2ccPA treatment for 4 weeks increased high-density lipoprotein cholesterol (HDL-C) levels in mice fed a high-fat diet.

Figure 2. Expression of atherogenic target genes in carotid arteries of mice in response to 4 weeks of 2ccPA treatment. 2ccPA inhibited expression of atherogenic target genes in apolipoprotein $\mathrm{E}$ knockout mice. (A) Carotid artery lesions of 4-weeks old mice on high-fat diet after treatment with or without 2ccPA. (B) Real-time PCR measurement of mRNA of genes encoding CD36, PPAR $\gamma$, FABP4, and VEGF in the carotid arteries of mice after treatment with or without $2 \mathrm{ccPA}$ for 4 weeks. Gene expression levels were normalized to that of the $\beta$-actin gene and are expressed as mean $\pm \operatorname{SEM}(\mathrm{n}=3), * * \mathrm{P}<0.01$.

Figure 3. Expression of genes related to cytokines and chemokines in the carotid arteries of mice treated with 2ccPA for 4 weeks. (A) 2ccPA inhibited expression of atherogenic target genes in apolipoprotein $\mathrm{E}$ knockout mice. A) Real-time PCR measurement of mRNA of genes encoding IL-6, RANTES, MCP-1, and TNF- $\alpha$ in carotid arteries of mice after treatment with or without $2 \mathrm{ccPA}$ for 4 weeks. Gene expression levels were normalized to that of the $\beta$-actin gene and are expressed as mean $\pm \operatorname{SEM}(\mathrm{n}=3), * * \mathrm{P}<0.01$.

Figure 4. Comparison of endogenous class I HDAC expression in carotid arteries in mice (A) Real-time PCR was used to measure Hdac2, and Hdac3 mRNA expression in carotid arteries using specific primers for each Hdac gene. The relative expression of each Hdac gene isoform was calculated by normalization to the expression of the $\beta$-actin gene and was expressed as the mean $\pm \operatorname{SEM}(\mathrm{n}=3)$. $* * \mathrm{P}<0.01$. 
A

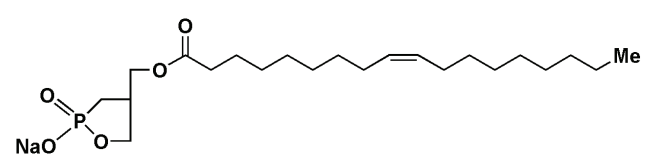

2ccPA

C

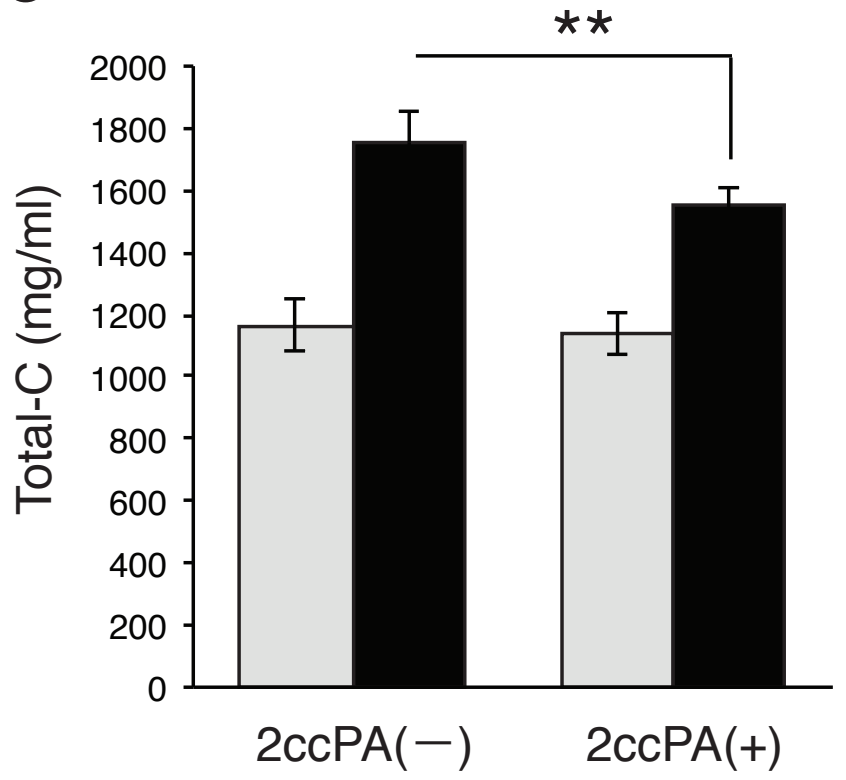

E

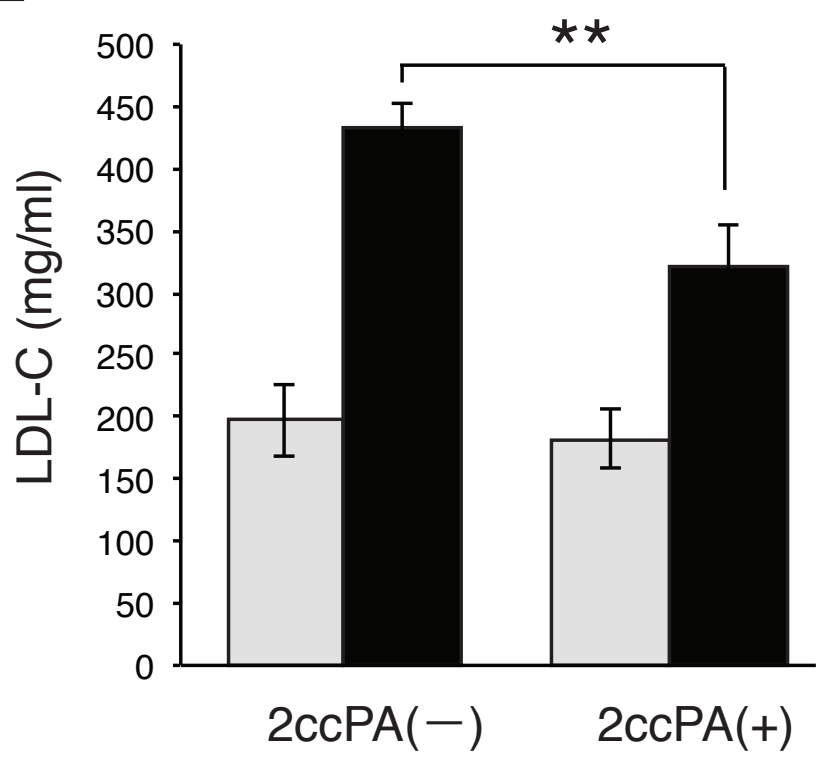

B

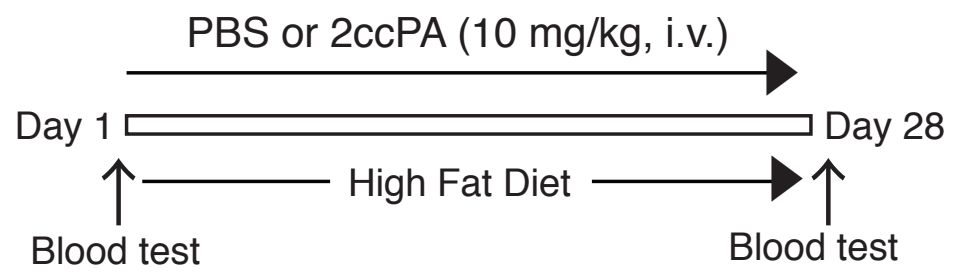

D

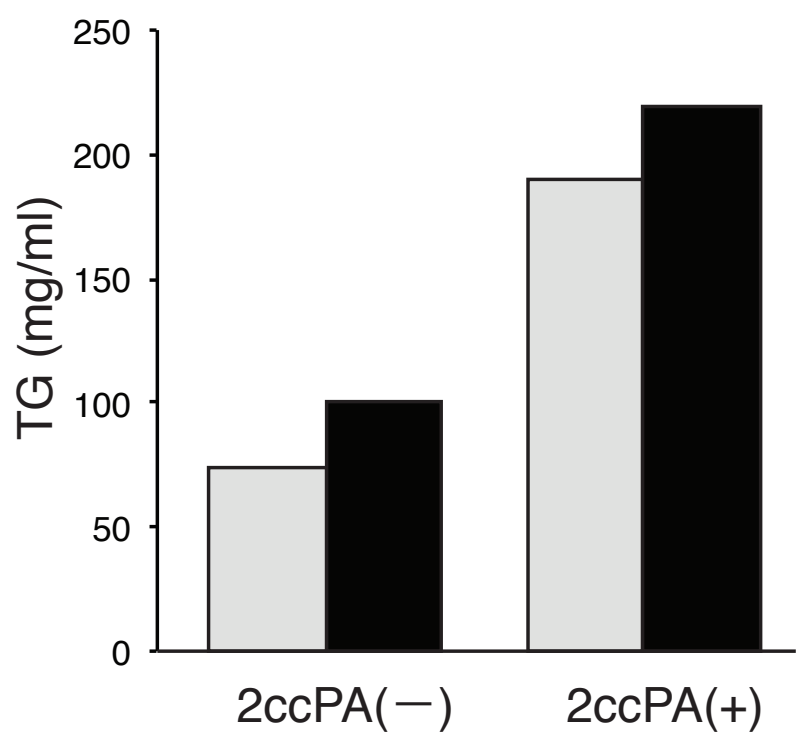

F

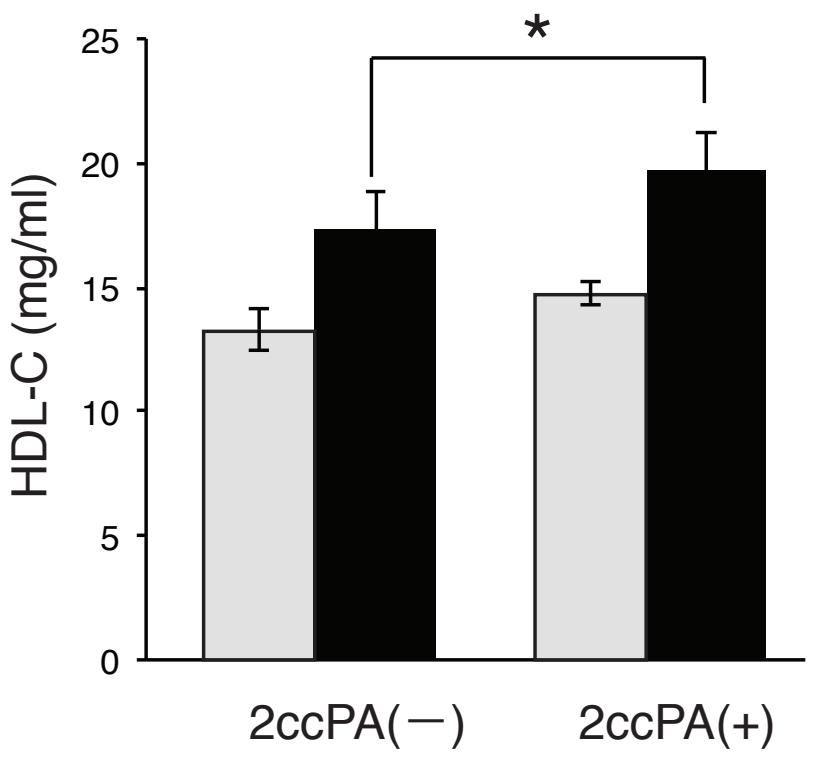

Day 1 Day 28

Fig. 1 

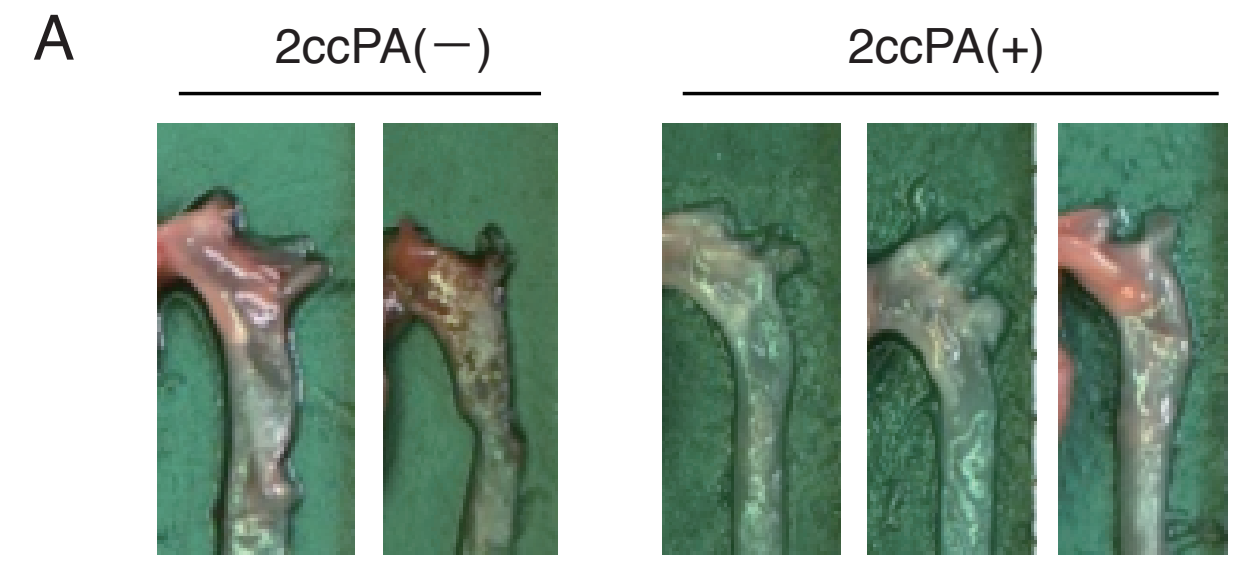

B

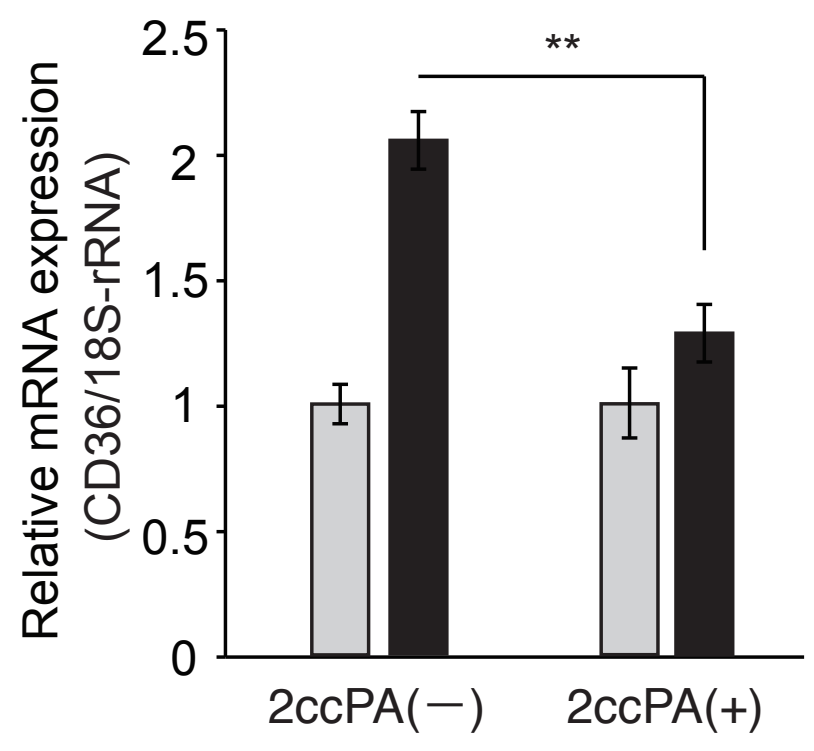

D

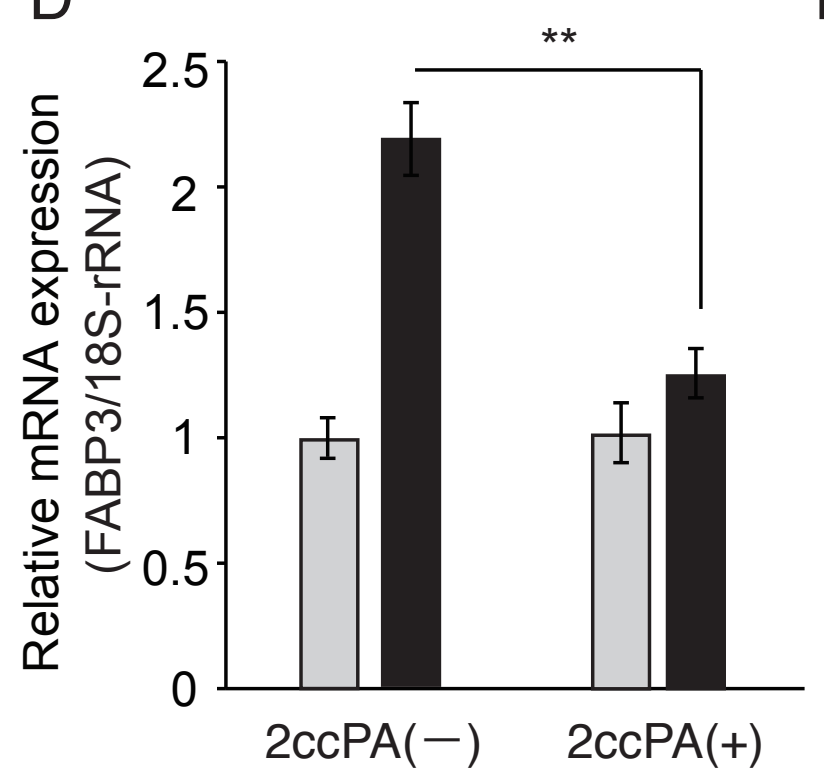

C

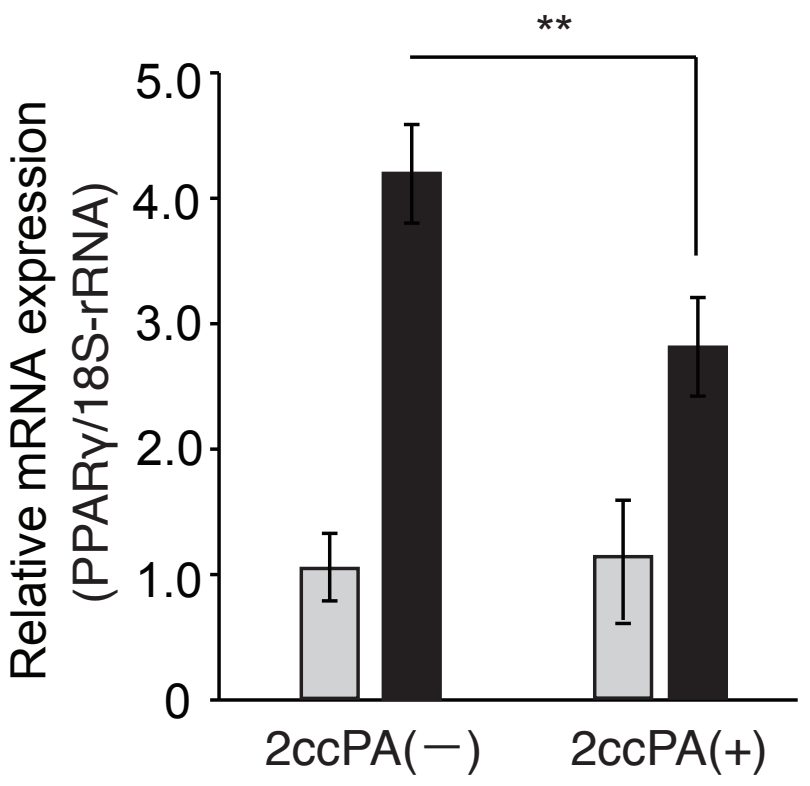

E

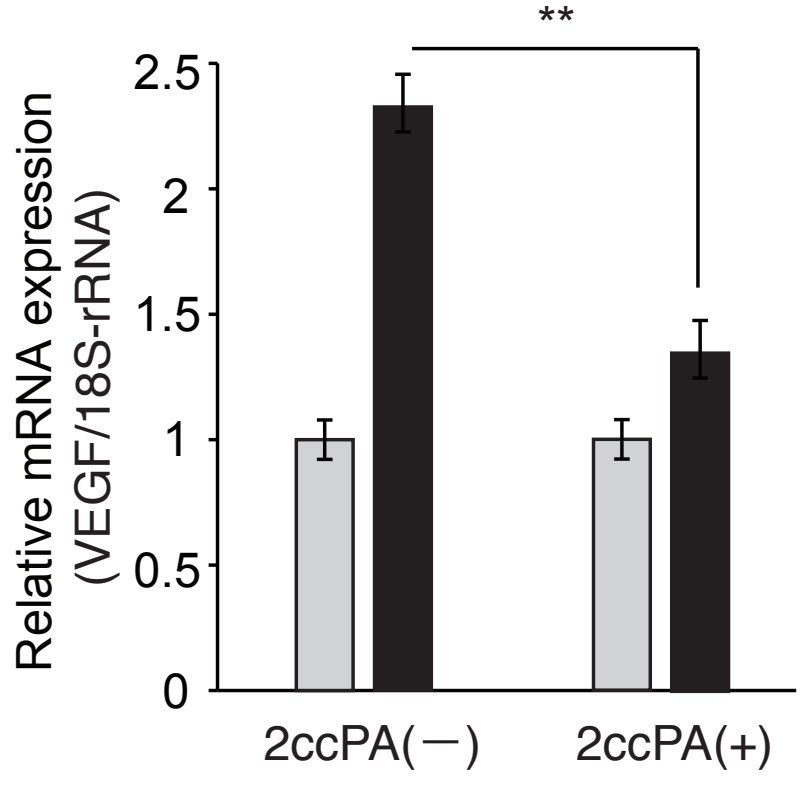

Fig. 2 
A

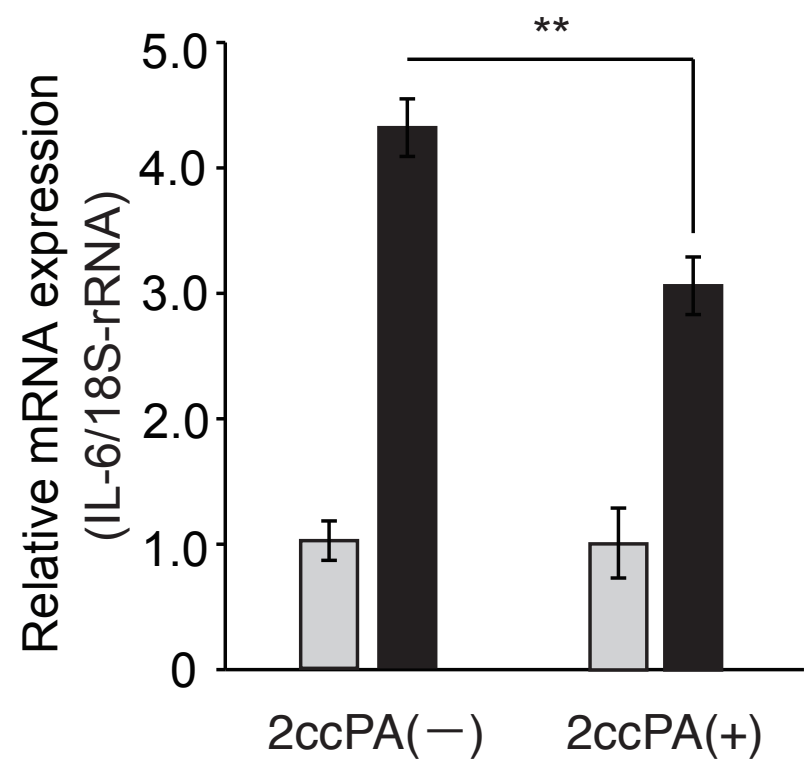

C

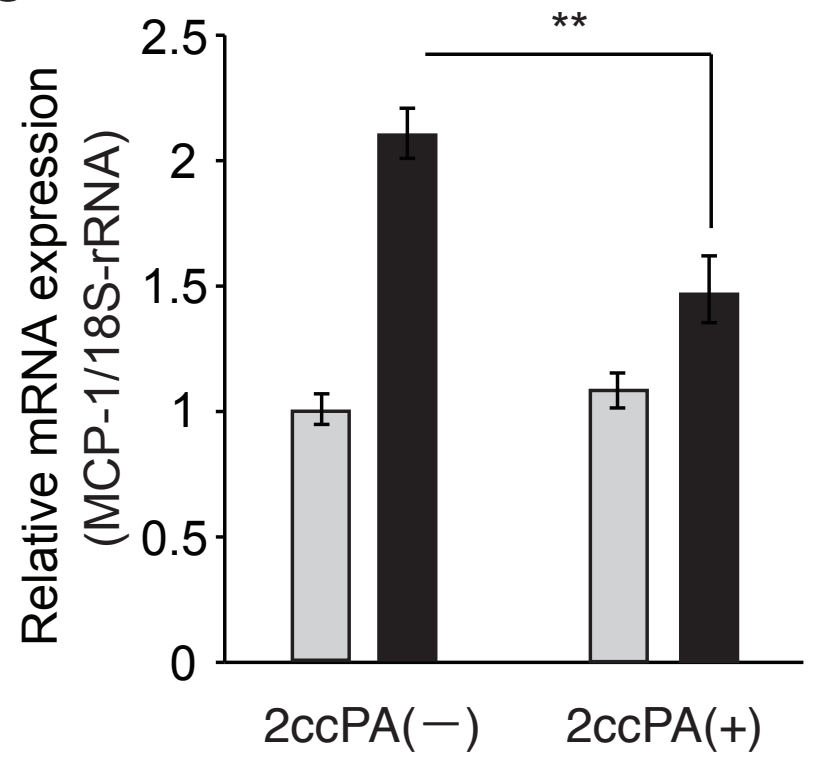

B

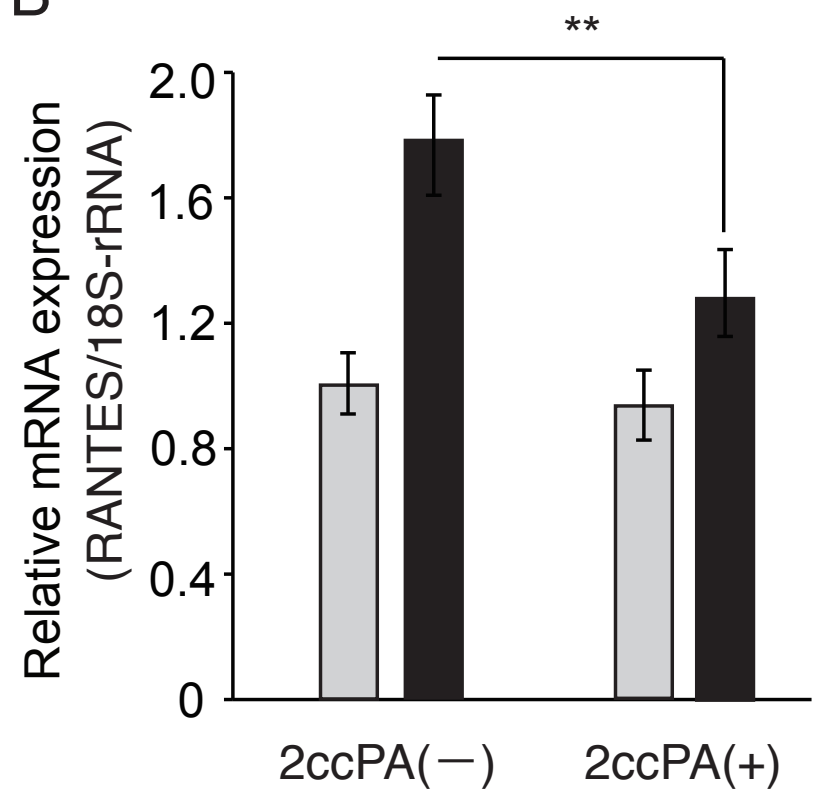

D

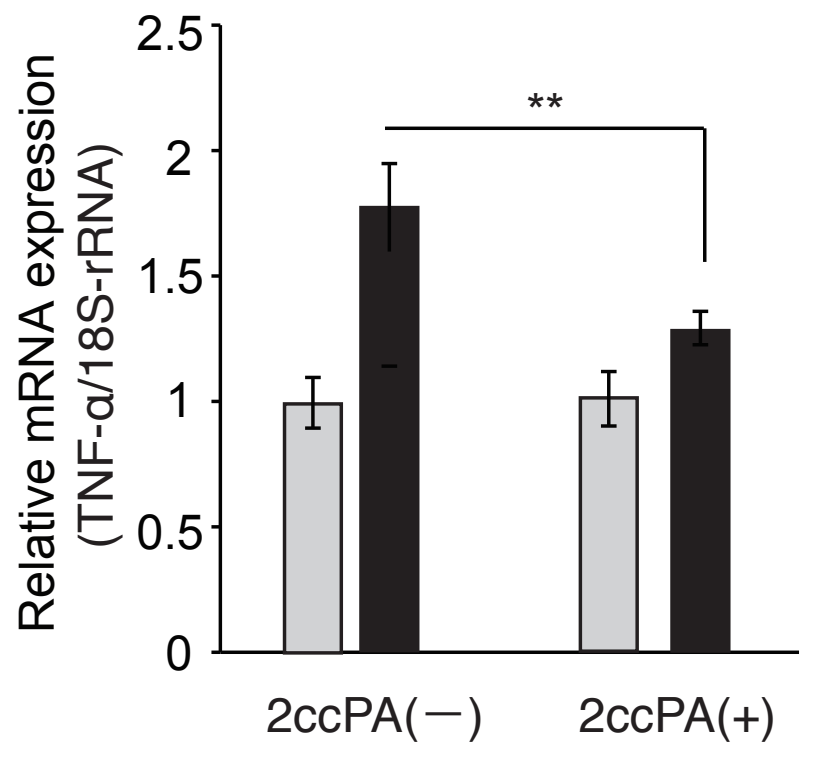

$\square$ Day $1 \quad$ Day 28

Fig. 3 

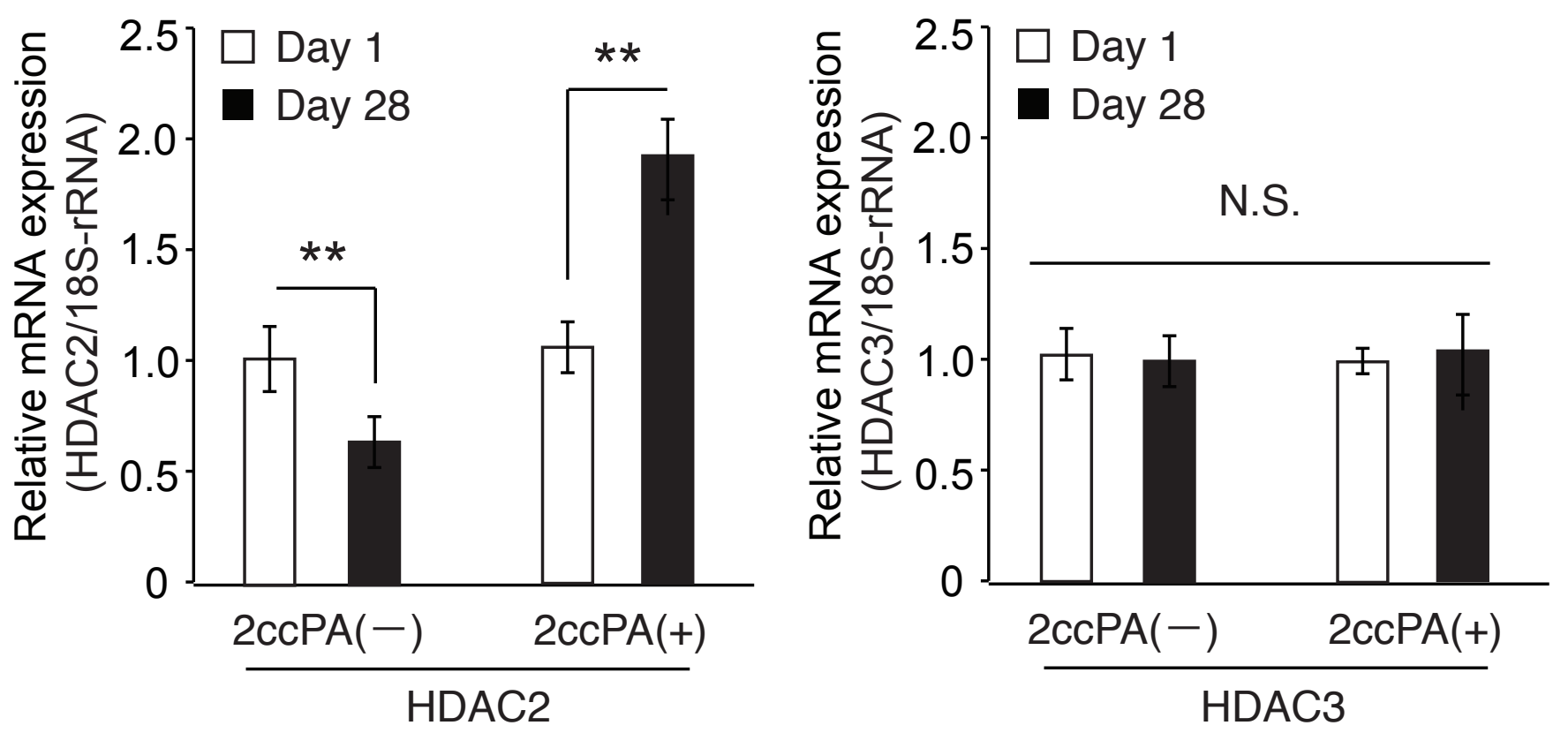